\title{
Charm production in DIS and the measurement of $\mathrm{F}_{2}{ }^{\mathrm{cc}}$ at ZEUS
}

\section{DIS 2009}

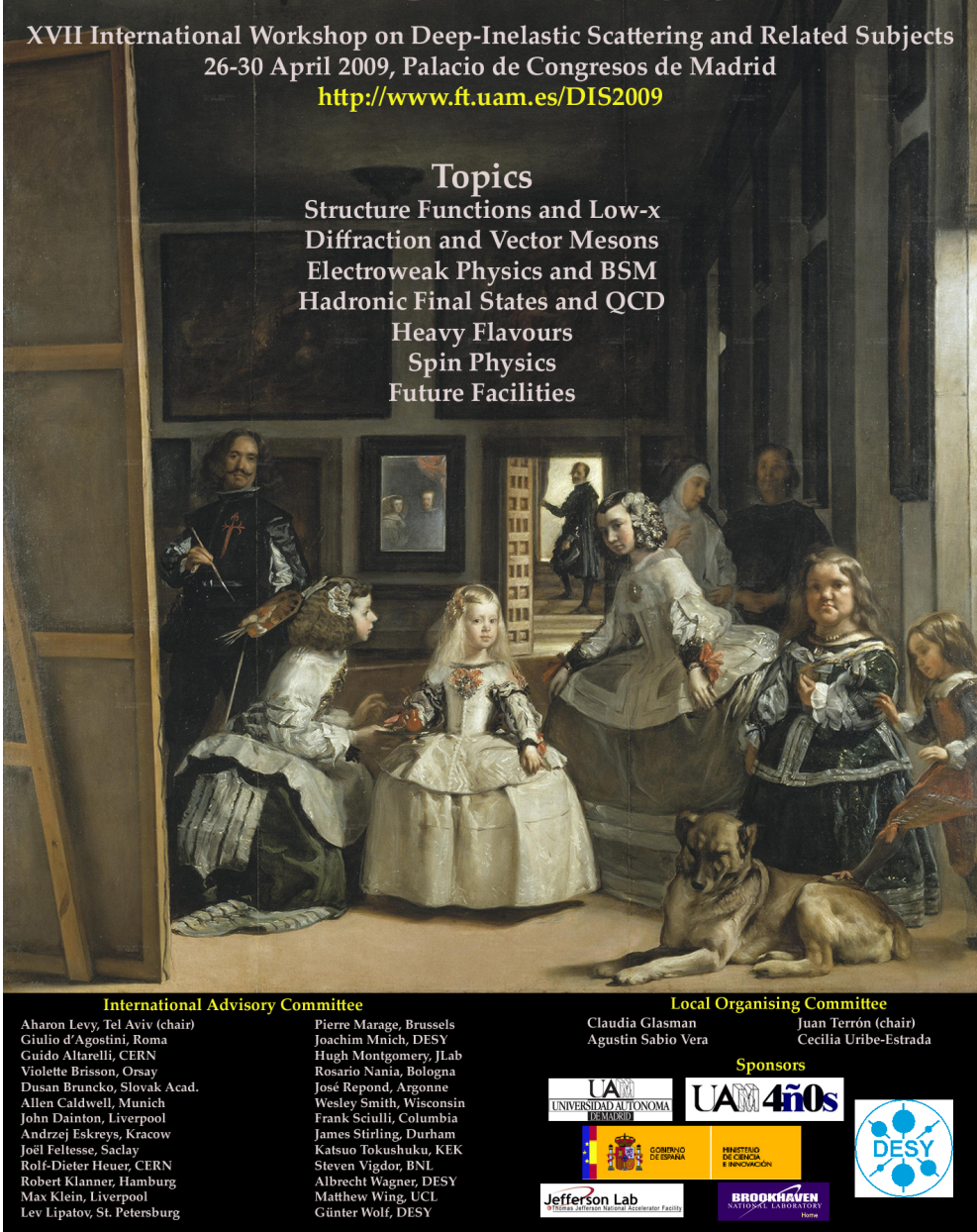

Philipp Roloff

(DESY \& University of Hamburg)

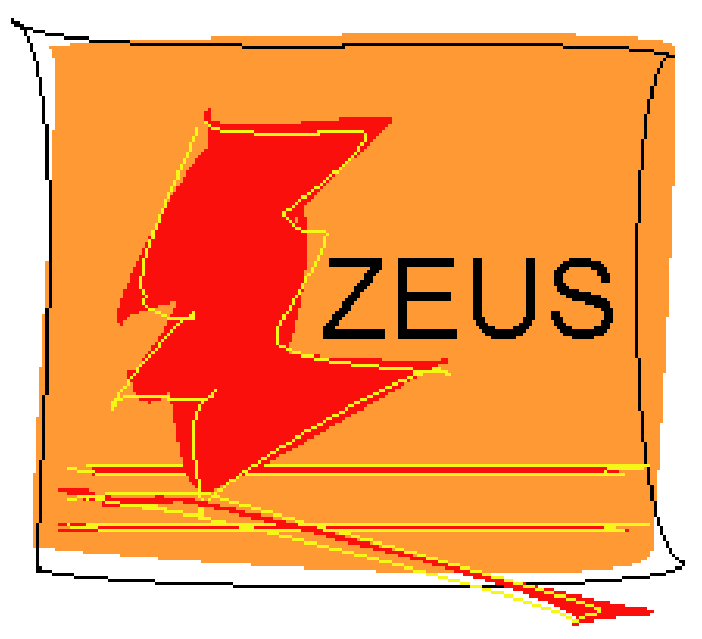

XVII International Workshop on Deep-Inelastic Scattering and Related Subjects DIS 2009, 26-30 April 2009, Madrid 


\section{Outline}

- Introduction

- Measurement of $\mathrm{D}^{ \pm}$and $\mathrm{D}^{0}$ production (DESY-08-201)

- Charm from decays into muons

(DESY-09-056)

- Extraction of $\mathrm{F}_{2}^{\mathrm{cc}}$

- Summary 


\section{Charm production at HERA}

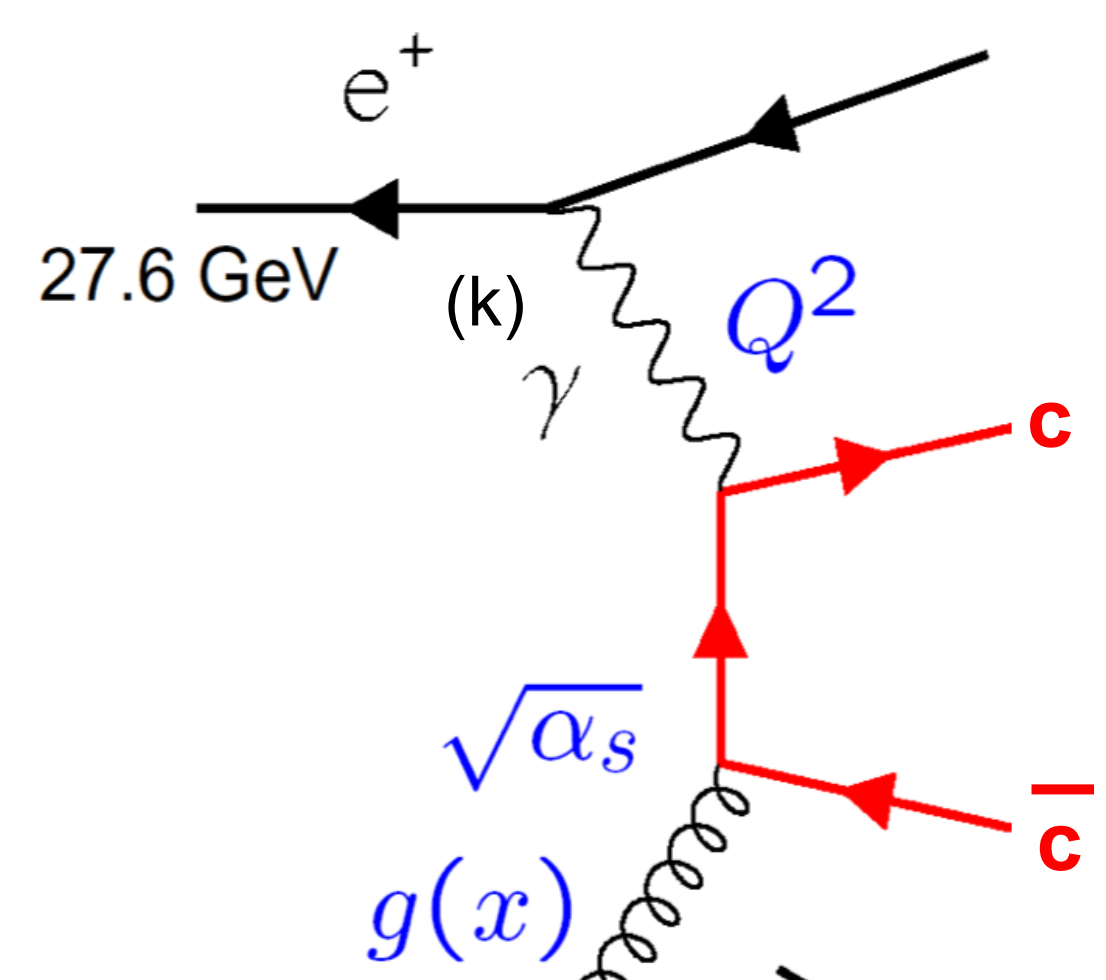

$920 \mathrm{GeV}$

$\mathrm{p}$
- Dominant process for charm production in DIS $\left(Q^{2}>\right.$ a few $\left.\mathrm{GeV}^{2}\right)$ : Boson-Gluon-Fusion (BGF)

- This process is directly sensitive to the gluon content of the proton

- Multiple hard scales:

$\mu^{2}=m^{2}(c), p_{T}^{2}(c), Q^{2}$

- The double differential

cross section for the production of open charm can be written as:

$$
\frac{d^{2} \sigma^{c \bar{c}}\left(x, Q^{2}\right)}{d x d Q^{2}}=\frac{2 \pi \alpha^{2}}{x Q^{4}}\left\{\left[1+(1-y)^{2}\right] F_{2}^{c \bar{c}}\left(x, Q^{2}\right)-y^{2} F_{L}^{c \bar{c}}\left(x, Q^{2}\right)\right\}
$$




\section{pQCD Treatment of charm (and beauty) production in DIS}

\section{Massive, FFNS:}

$\mathrm{c}$ and b produced dynamically (not part of proton or photon)

- $c$, b massive

- Neglects $\left[\alpha_{s} \ln \left(\mu^{2} / m^{2}\right)\right]^{n}$

- Valid at threshold

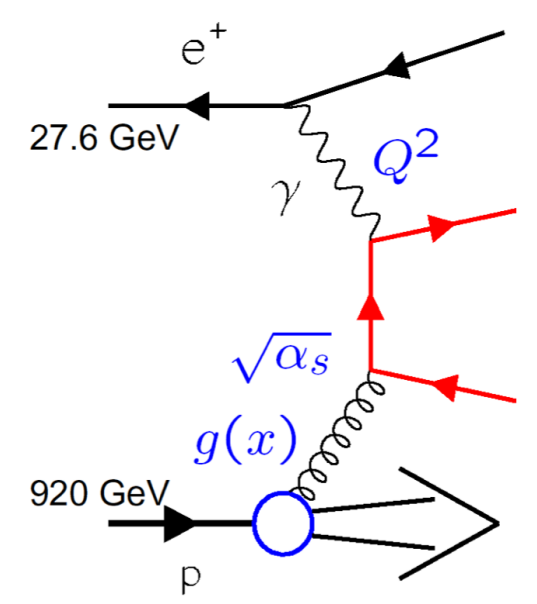

Massless, ZM-VFNS:

$c$ and $b$ massless partons in proton and photon

- c, b massless

- Resums $\left[\alpha_{s} \ln \left(\mu^{2} / m^{2}\right)\right]^{n}$

- Valid for $\mu^{2}>\mathrm{m}^{2}$

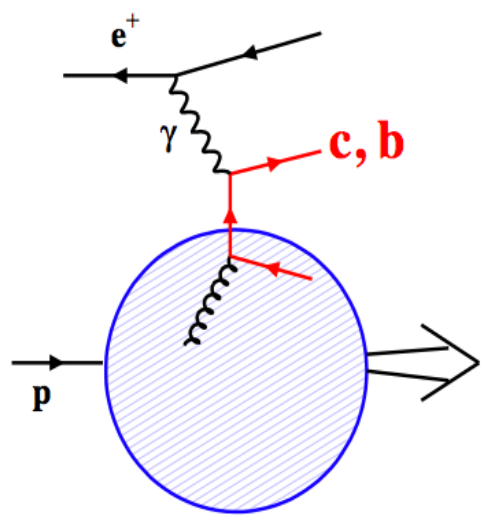

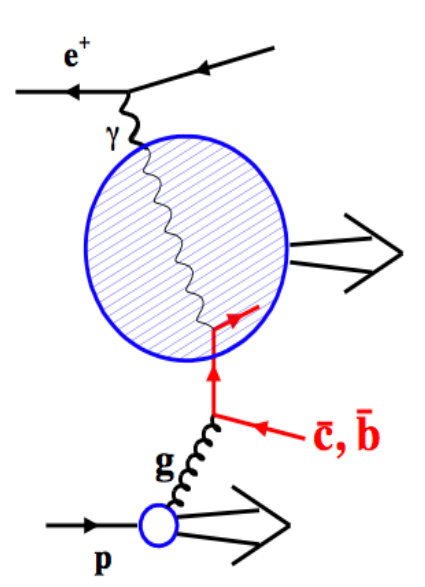

Variable Flavour Number Scheme, (GM)-VFNS:

Interpolates / matches between both approaches

- Massive at low $\mathrm{Q}^{2}$, massless at high $\mathrm{Q}^{2}$ 


\section{Tracking at ZEUS in HERA II}

\section{ZEUS Tracking}

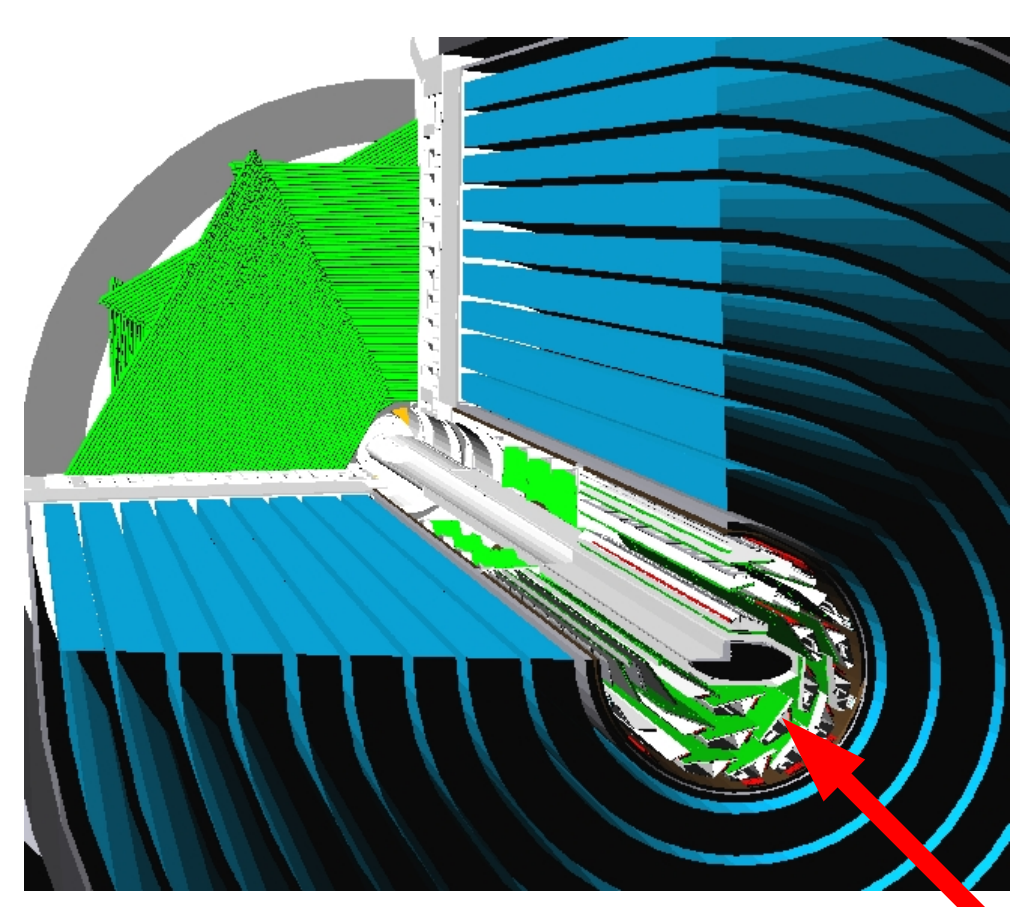

- For HERA II ZEUS was equipped with a silicon Micro Vertex Detector (MVD)

- Barrel region (BMVD):

3 layers of strip sensors

- Forward region (FMVD):

4 wheels of strip sensors

- BMVD resolution after alignment: $25 \mu \mathrm{m}$

- CTD (wire chamber) + BMVD impact parameter resolution: $100 \mu \mathrm{m}$ (Intermediate state of the data, better precision has been achieved)

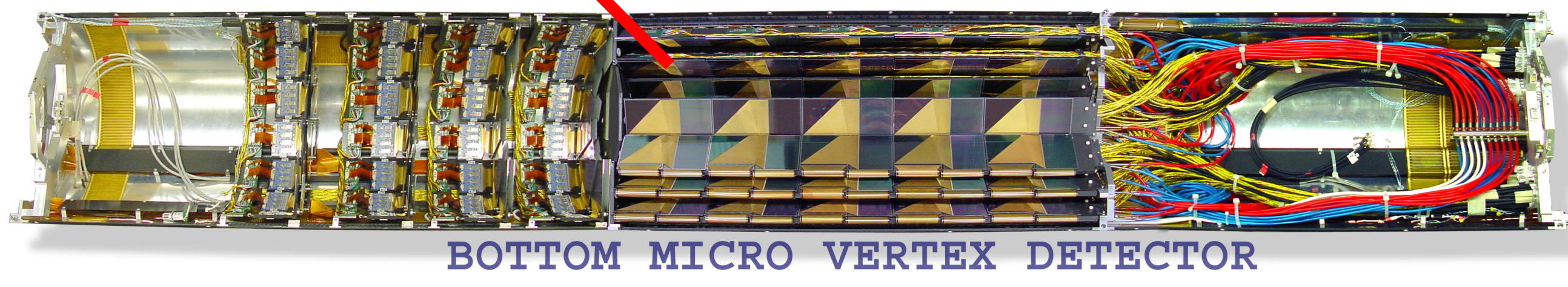




\section{Measurement of $D^{ \pm}$and $D^{0}$ production}

- Charmed mesons are tagged in the decays $\mathrm{D}^{+} \rightarrow \mathrm{K}^{-} \mathrm{T}^{+} \pi^{+}$(+C.C.) and $\mathrm{D}^{0} \rightarrow \mathrm{K}^{-} \pi^{+}(+\mathrm{C}$.c. $)$

- The secondary vertices of these decays can be reconstructed

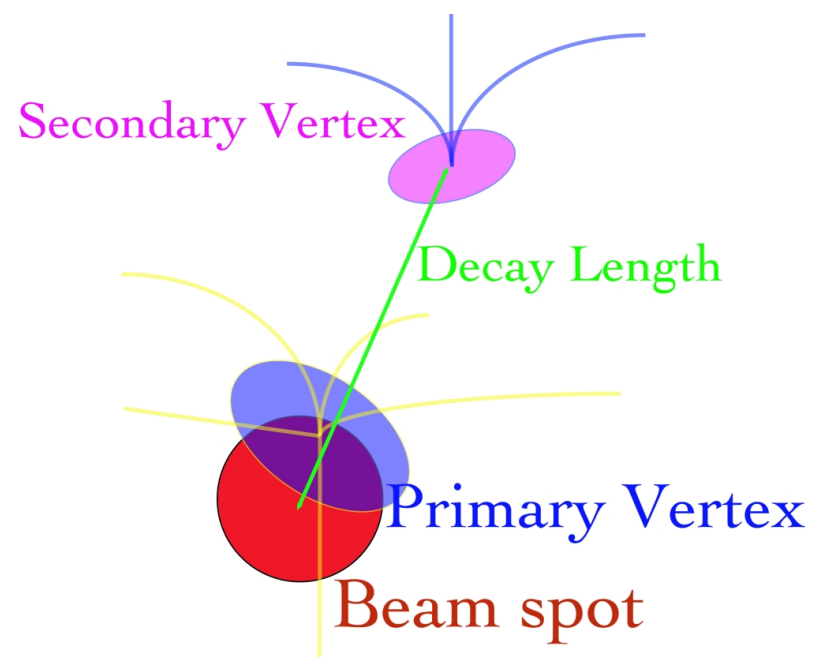

- $\mathrm{L}_{\mathrm{XY}}: 2 \mathrm{D}$ distance between the secondary vertex and the primary interaction point projected onto the D meson momentum vector

- $\mathrm{S}_{1}=\mathrm{L}_{X Y} / \sigma\left(\mathrm{L}_{X Y}\right)$ :

Positive for charm decays

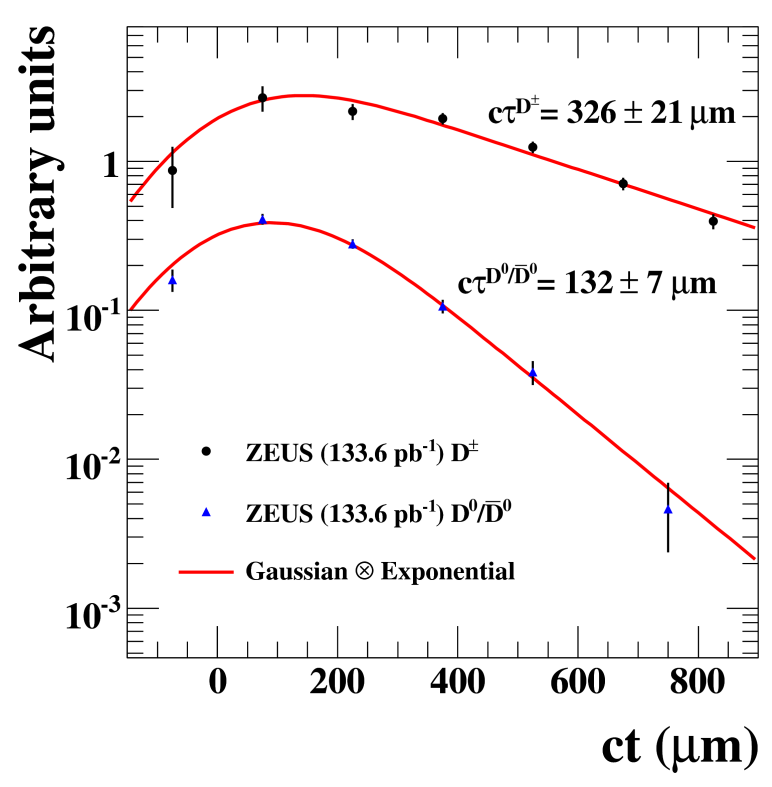




\section{Reconstruction and simulation of secondary vertices}

- Cuts for $\mathbf{D}^{ \pm}$: 3 degrees of freedom $\mathrm{S}_{1}>3$

- Cuts for $\mathrm{D}^{0}$ :

$\chi^{2}$ (decay vertex) $<8$ for 1 degree of freedom $S_{1}>1$

- The dominant systematic uncertainty is related to the simulation of the MVD resolution

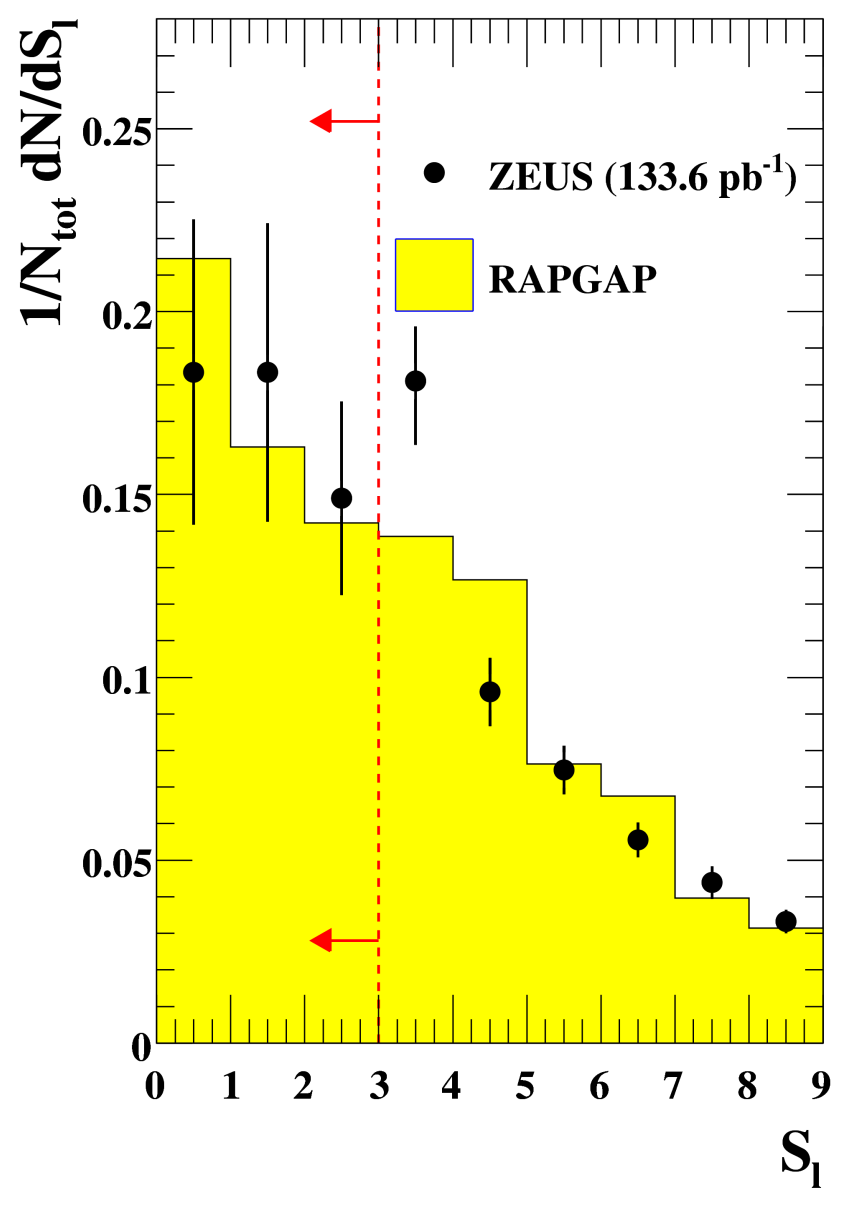




\section{$D^{ \pm}$and $D^{0}$ reconstruction and selection}

- Kinematic range:

$5<\mathrm{Q}^{2}<1000 \mathrm{GeV}^{2}$

$0.02<y<0.7$

$1.5<\mathrm{p}_{\mathrm{T}}(\mathrm{D})<15 \mathrm{GeV}$

$|\eta(D)|<1.6$

- Data sample:

$133.6 \mathrm{pb}^{-1}$

HERA II

e-p $(2004 / 05)$

- $\mathrm{D}^{0}$ mesons not

originating from $D^{* \pm}$

decays were used for the cross section measurement
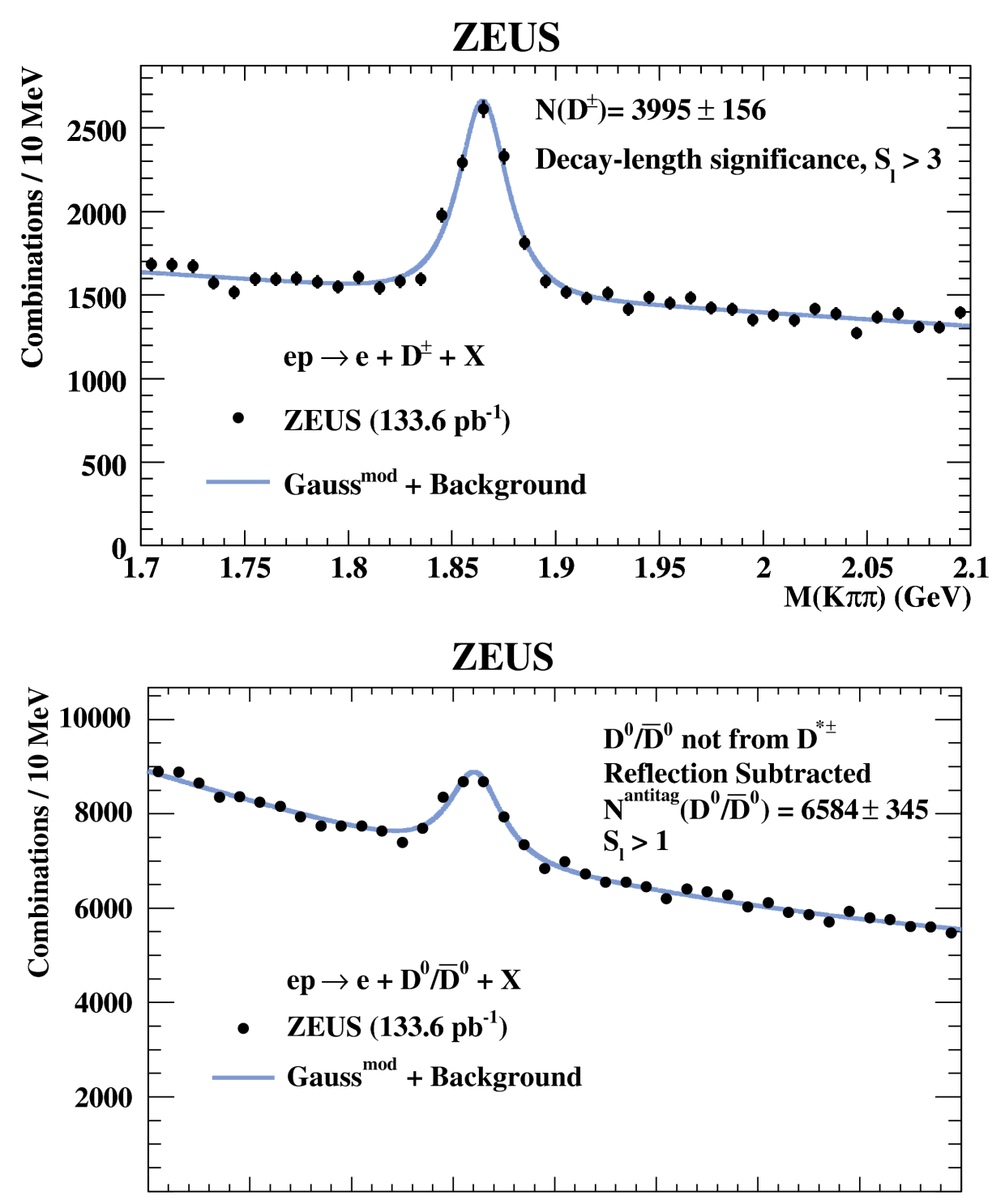


\section{$D^{ \pm}$cross sections: $Q^{2}$ and $x$}
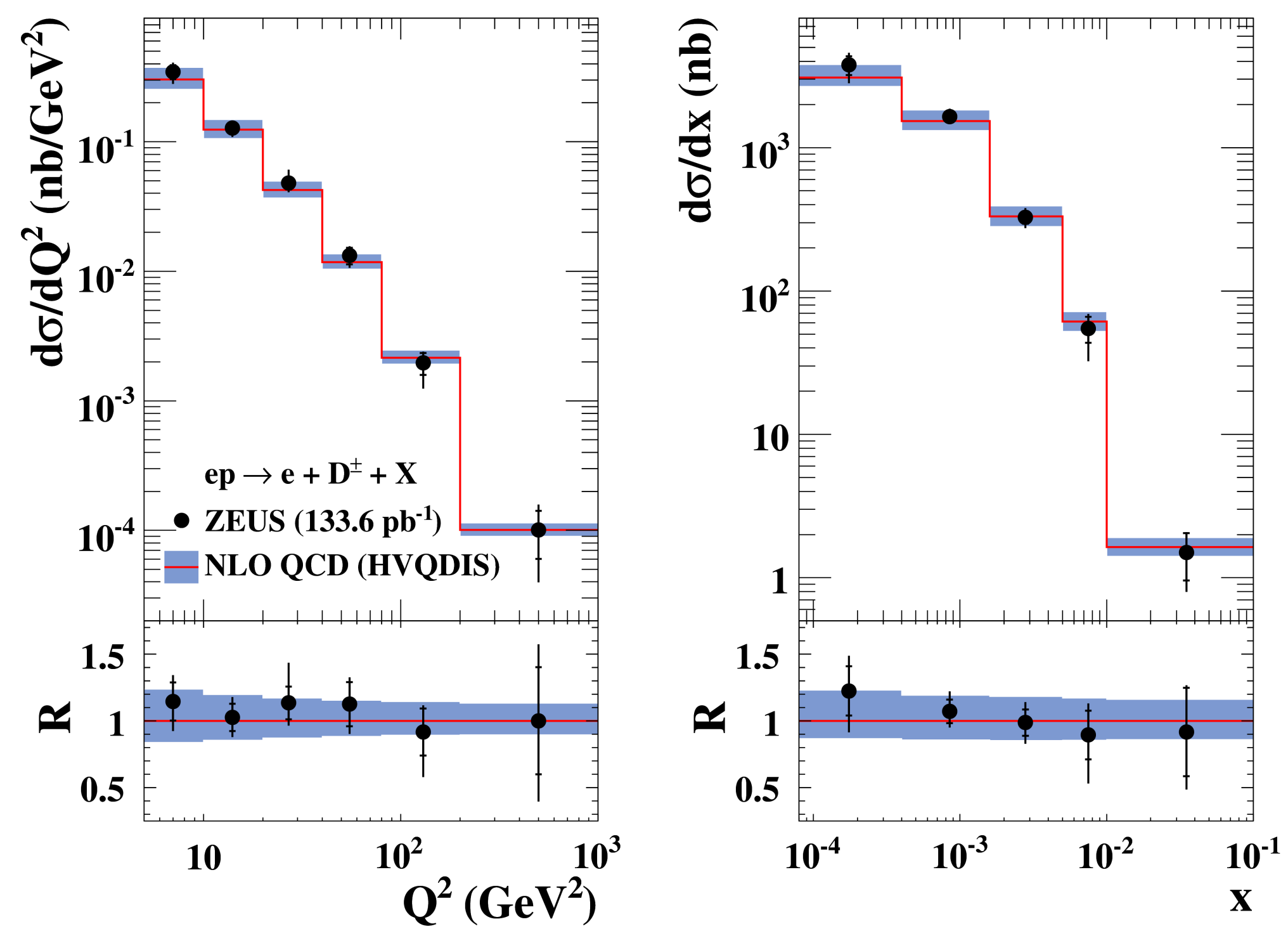

Good description by the HVQDIS NLO QCD prediction (FFNS) 


\section{$D^{ \pm}$cross sections: $p_{T}\left(D^{ \pm}\right)$and $\eta\left(D^{ \pm}\right)$}

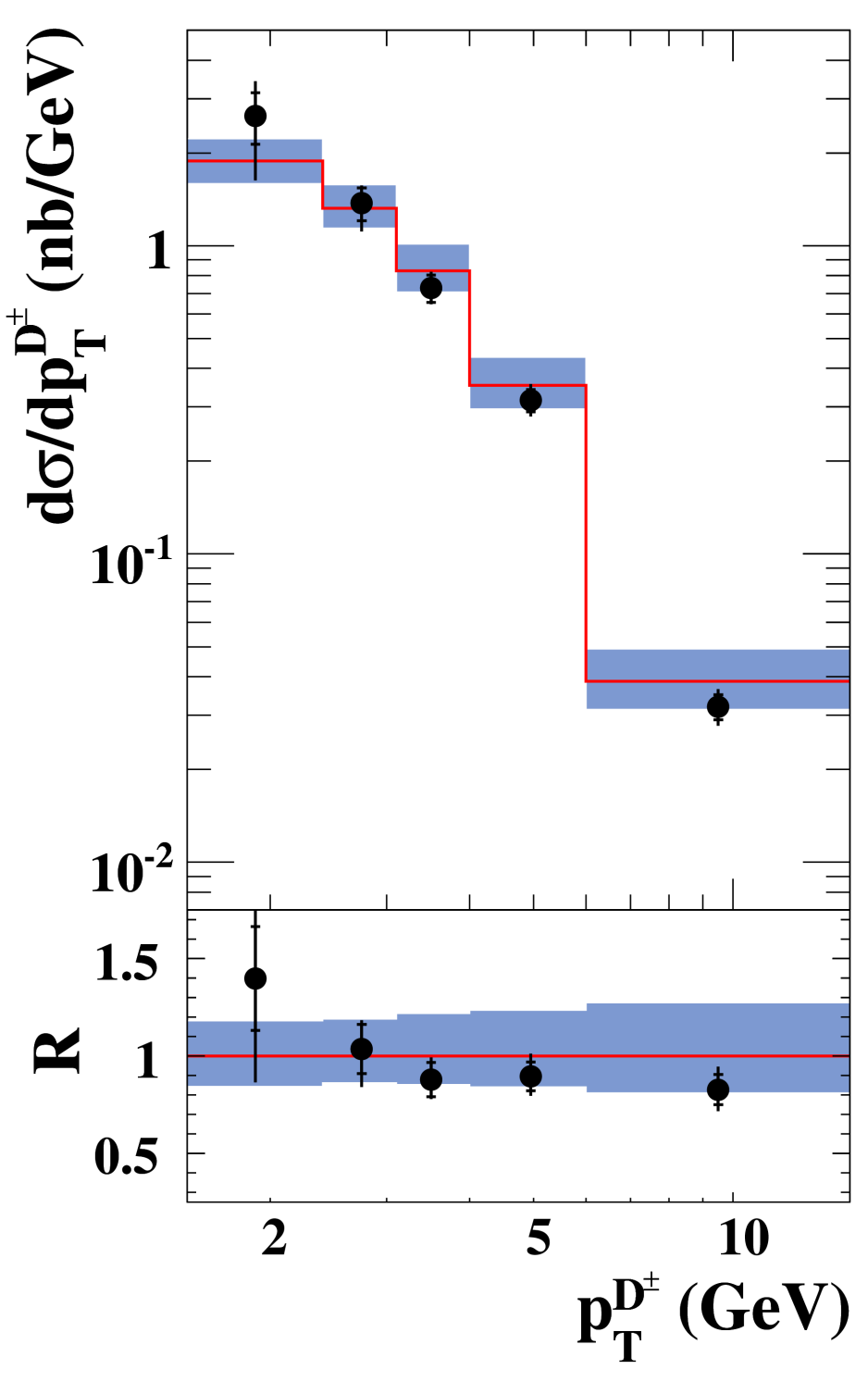

$28 / 04 / 2009$

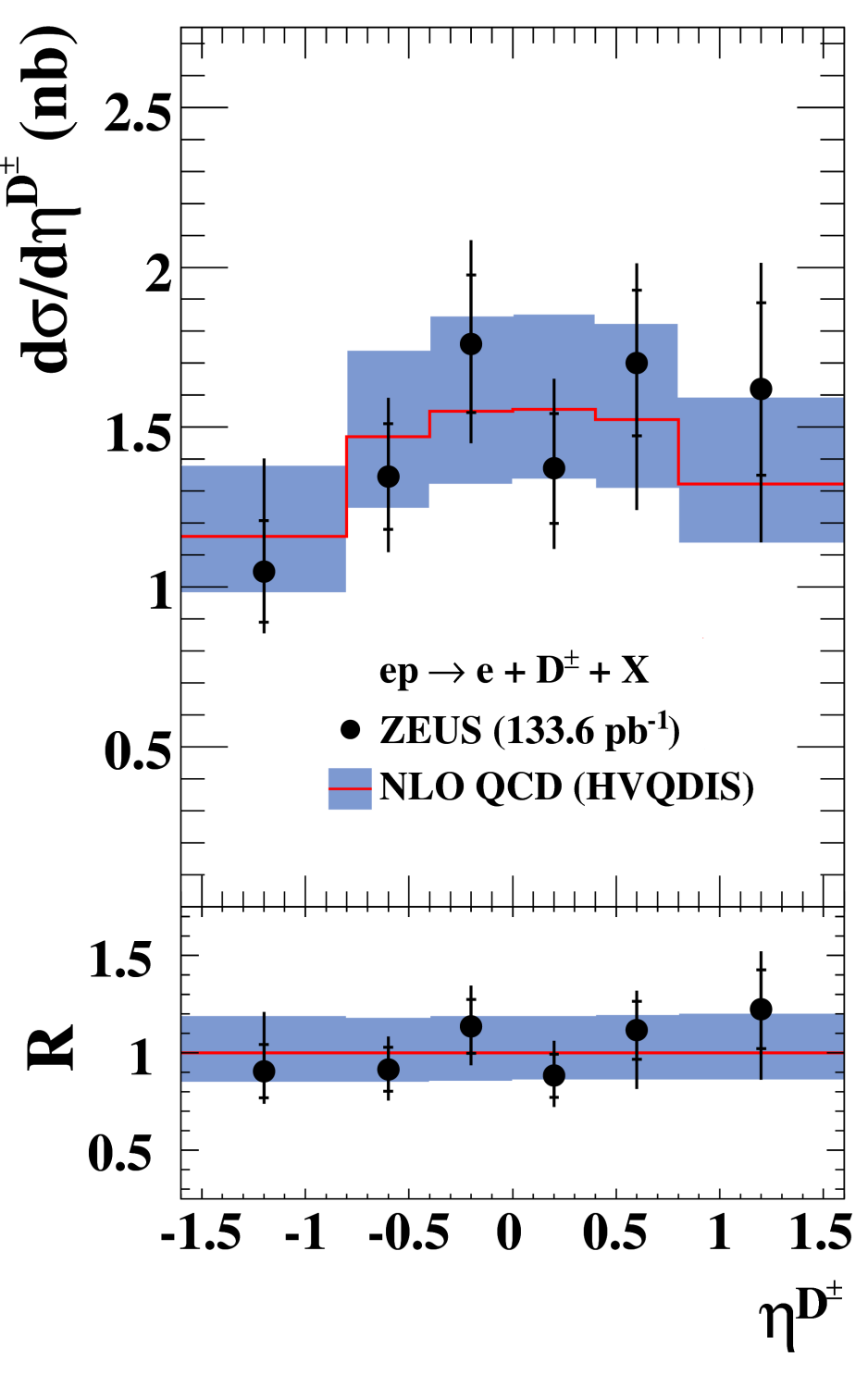

Good description by HVQDIS 


\section{$D^{0}$ cross sections: $Q^{2}$ and $x$}
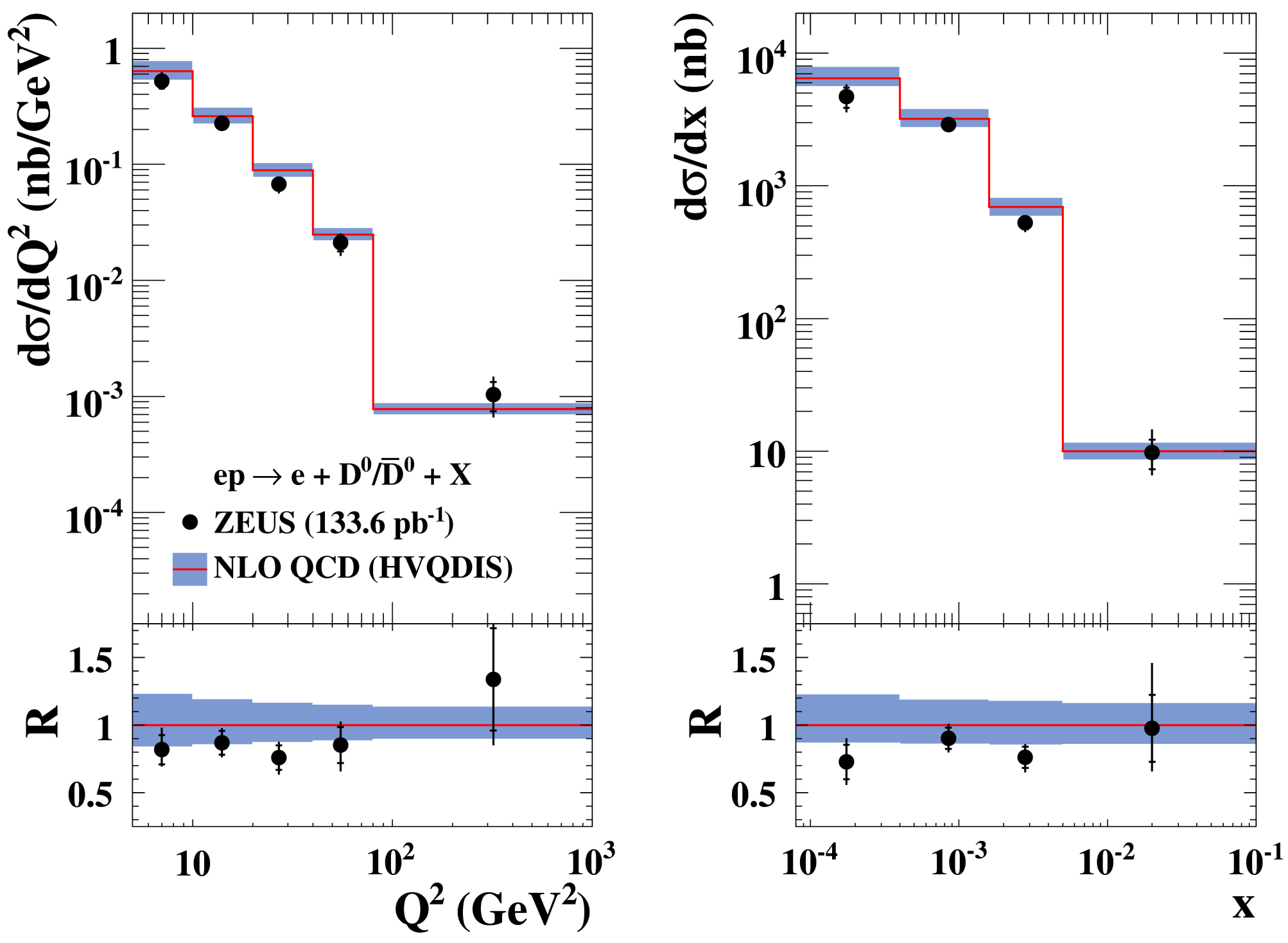

Measurements described by HVQDIS 


\section{$D^{0}$ cross sections: $p_{T}\left(D^{0}\right)$ and $\eta\left(D^{0}\right)$}

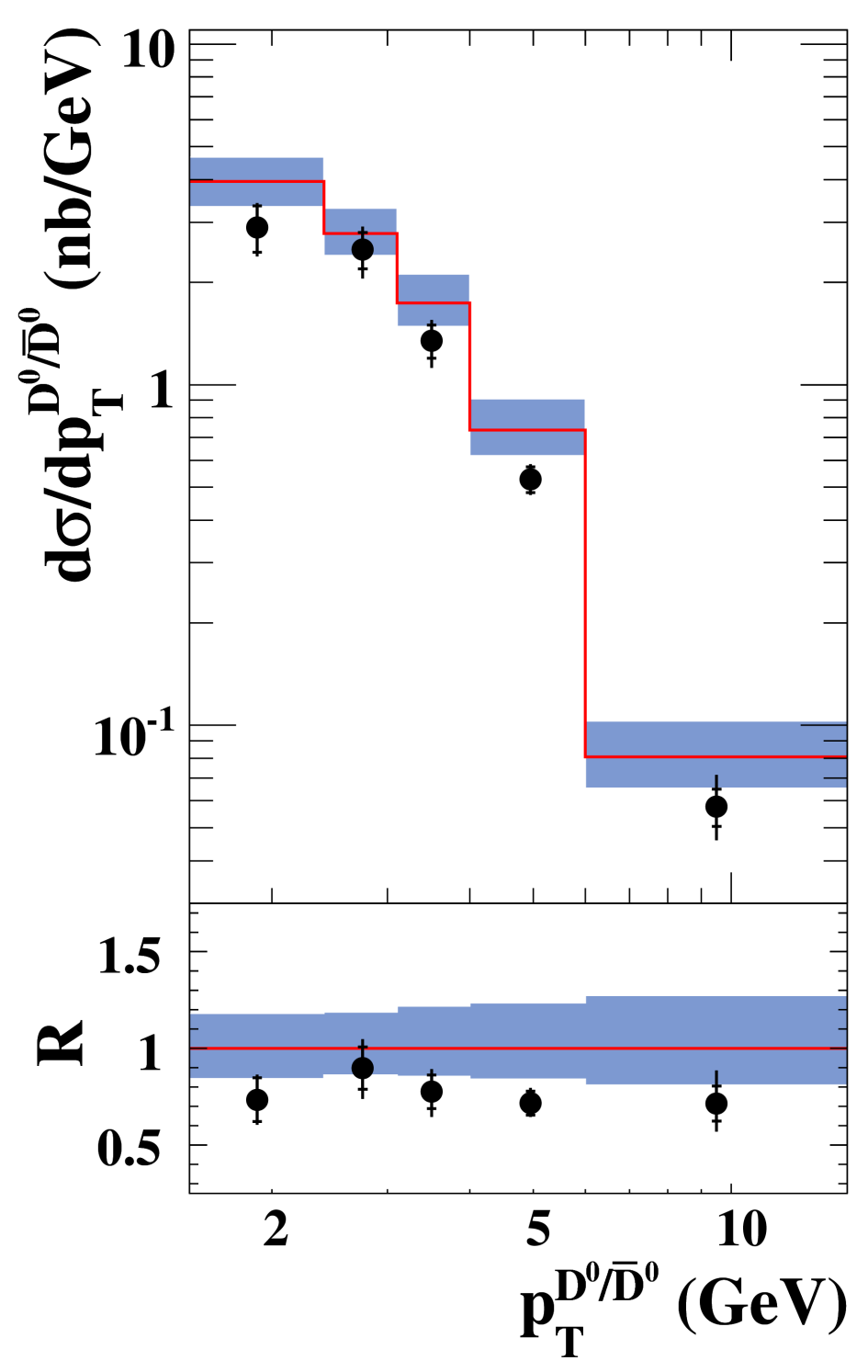

$28 / 04 / 2009$

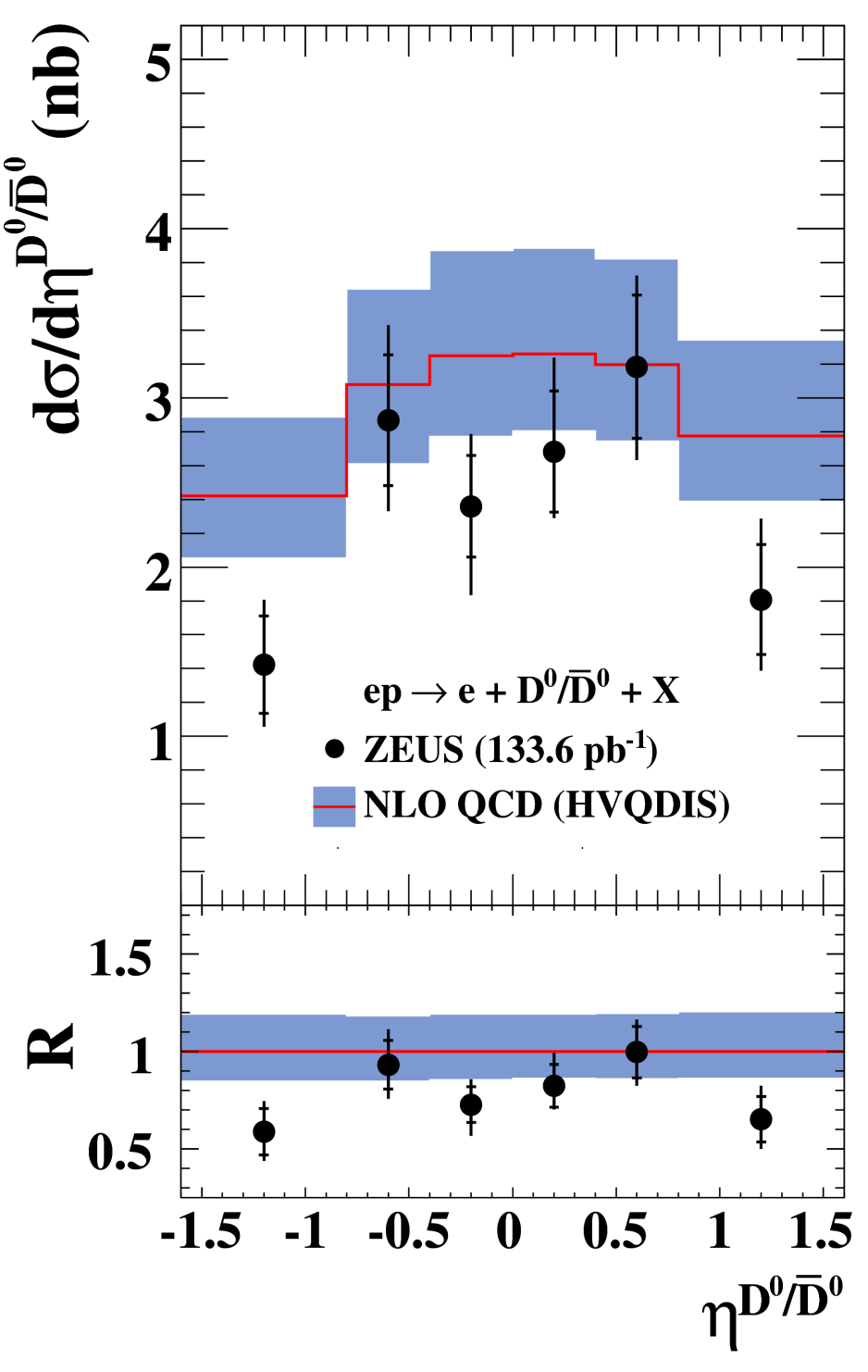

Measurements described by HVQDIS 


\section{Charm from decays into muons}

The fractions of muons from charm, beauty and LF events were obtained from a simultaneous fit of 3 discriminating variables:

- $\mathbf{p}_{\mathrm{T}}$ rel: the muon momentum component transverse to the jet axis

- $\delta$ : the impact parameter of the muon track

- $\mathbf{p}_{\mathrm{T}}^{\text {miss } \| \text { : }}$ : the missing transverse momentum parallel to the muon direction

$\rightarrow$ More details in talk by M. Bindi

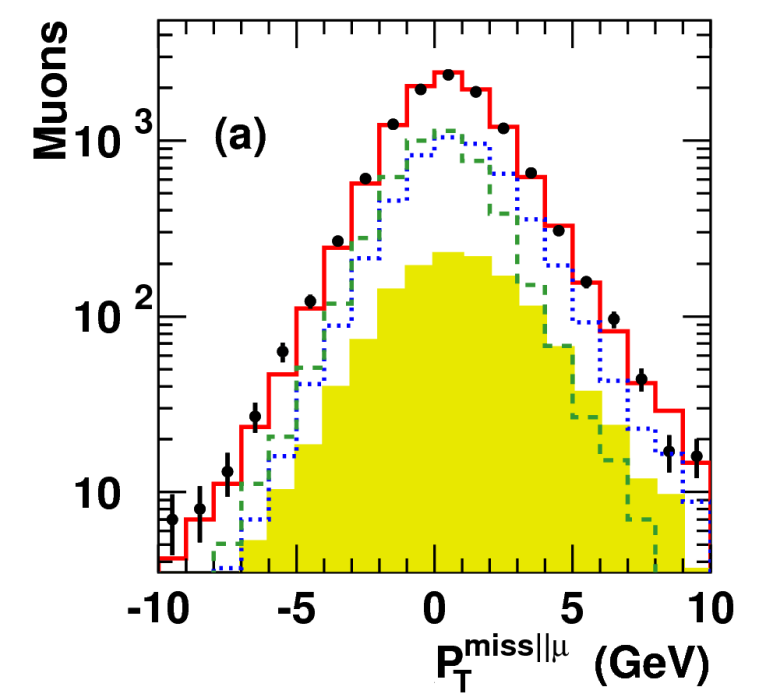

\section{ZEUS}

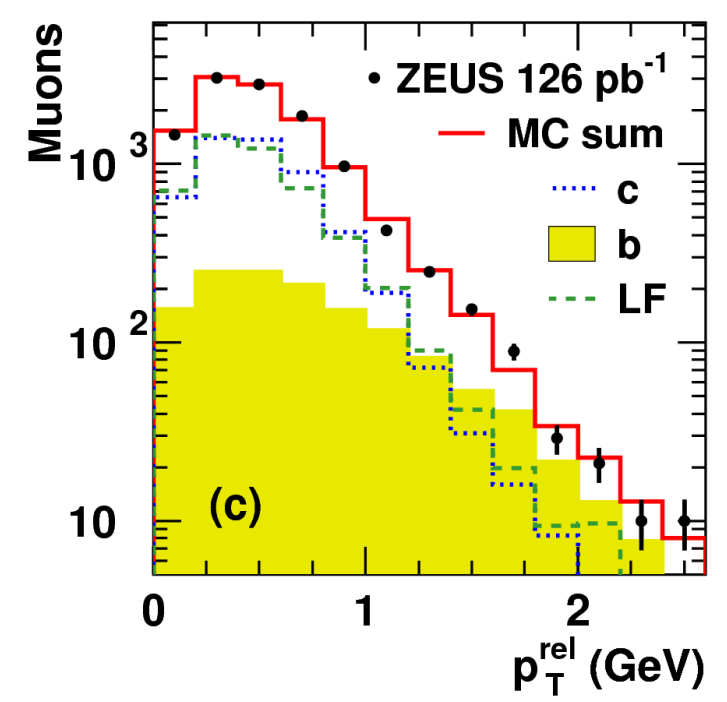

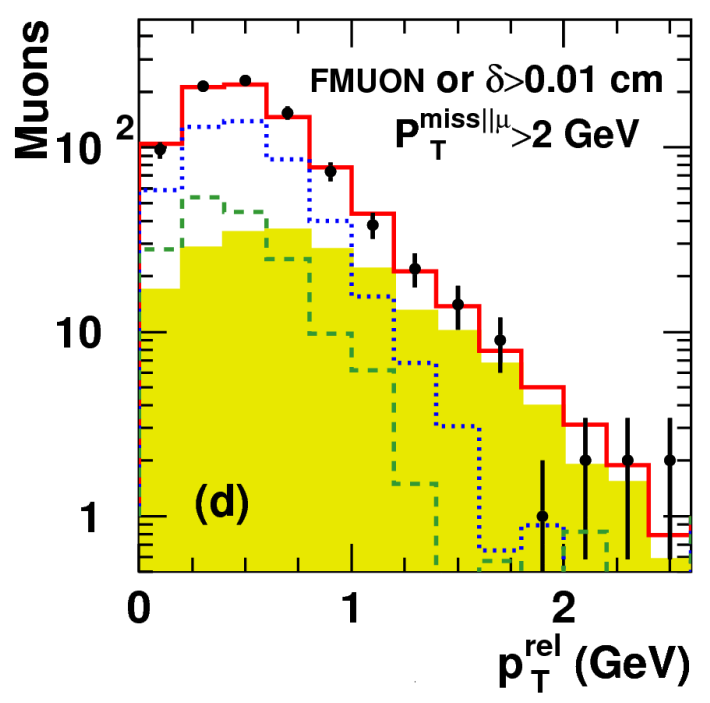




\section{Muon cross sections}

- Kinematic range:

$\mathrm{Q}^{2}>20 \mathrm{GeV}^{2}$

$0.01<y<0.7$

$\mathrm{p}_{\mathrm{T}}(\mu)>1.5 \mathrm{GeV}$

$-1.6<\eta(\mu)<2.3$

- Data sample: $126.0 \mathrm{pb}^{-1}$ HERA II e-p (2004/05)

- The charm cross sections are in good agreement with the HVQDIS predictions

$\rightarrow$ Beauty results in talk by M. Bindi

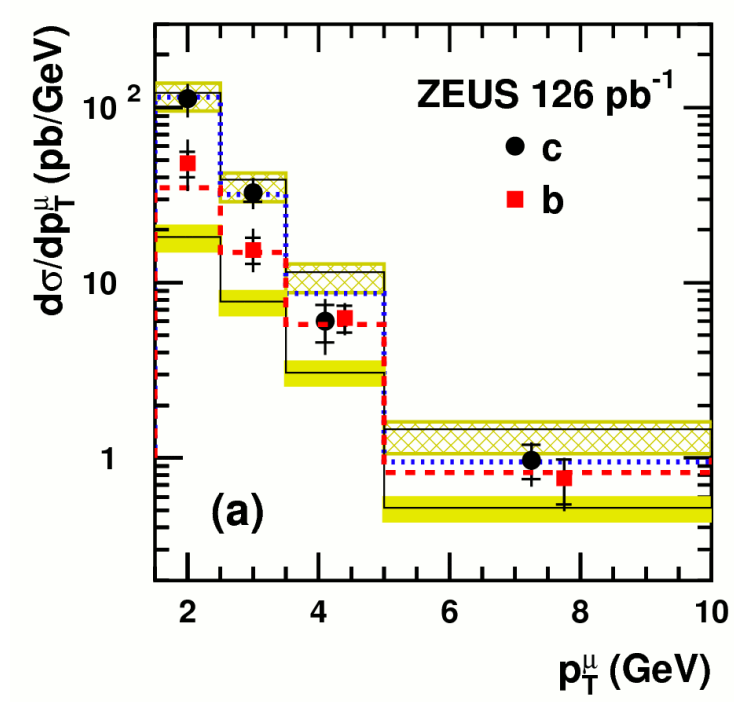

ZEUS
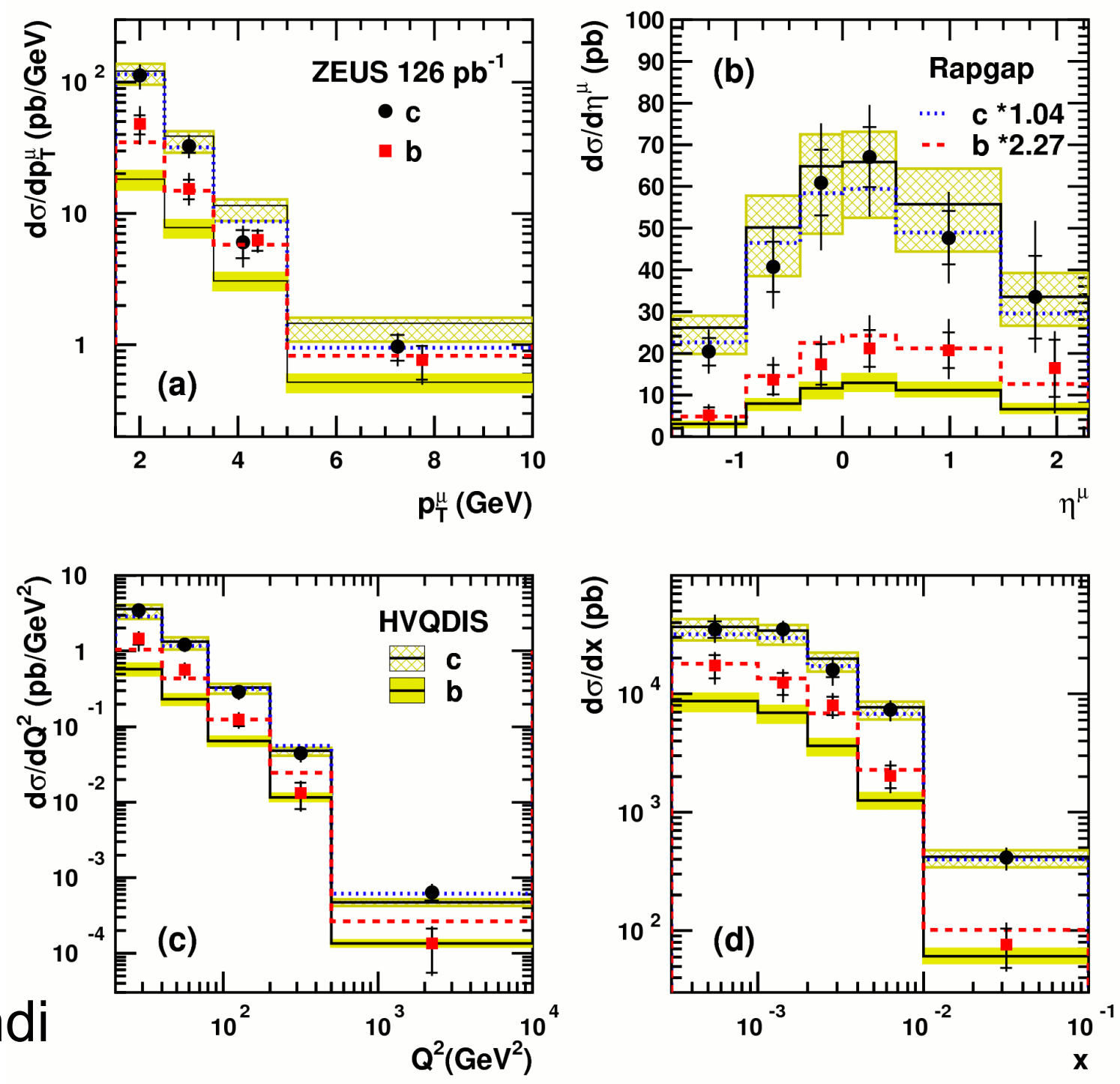


\section{Extraction of $\mathrm{F}_{2}^{\mathrm{cc}}$}

Extrapolation needed: Measured cross section in bin $\mathrm{i}$

$$
\begin{gathered}
F_{2, \text { meas }}^{c \bar{c}}\left(x_{i}, Q_{i}^{2}\right)=\frac{\sigma_{\text {meas }, i}}{\sigma_{\text {theo }, i}} \times F_{2, \text { theo }}^{c \bar{c}}\left(x_{i}, Q_{i}^{2}\right) \\
\begin{array}{c}
\text { Calculated using } \\
\text { HVQDIS (FFNS) }
\end{array} \\
\begin{array}{l}
\text { Calculated at NLO } \\
\text { in FFNS }
\end{array}
\end{gathered}
$$

Extrapolation factors for $D^{ \pm}$and $D^{0}$ measurement: 1.5 (at high $Q^{2}$ ) - $3.2\left(\right.$ at low $Q^{2}$ )

$\rightarrow$ Lower than in previous $D^{ \pm}$and $D^{0}$ analysis due to lower $p_{T}\left(D^{ \pm}, D^{0}\right)$ cut 


\section{$F_{2}{ }^{c c}$ from $D^{ \pm}$and $D^{0}$}
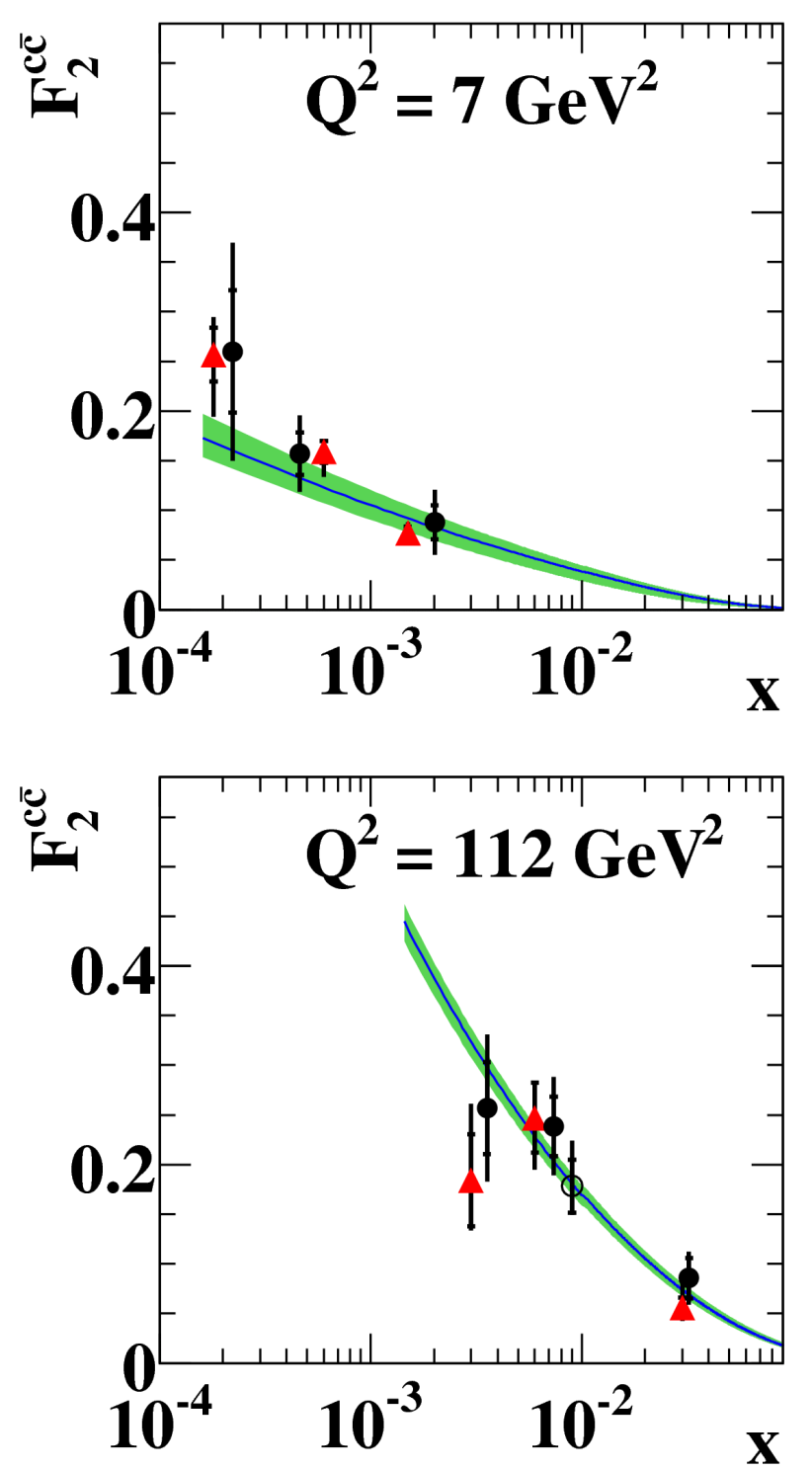

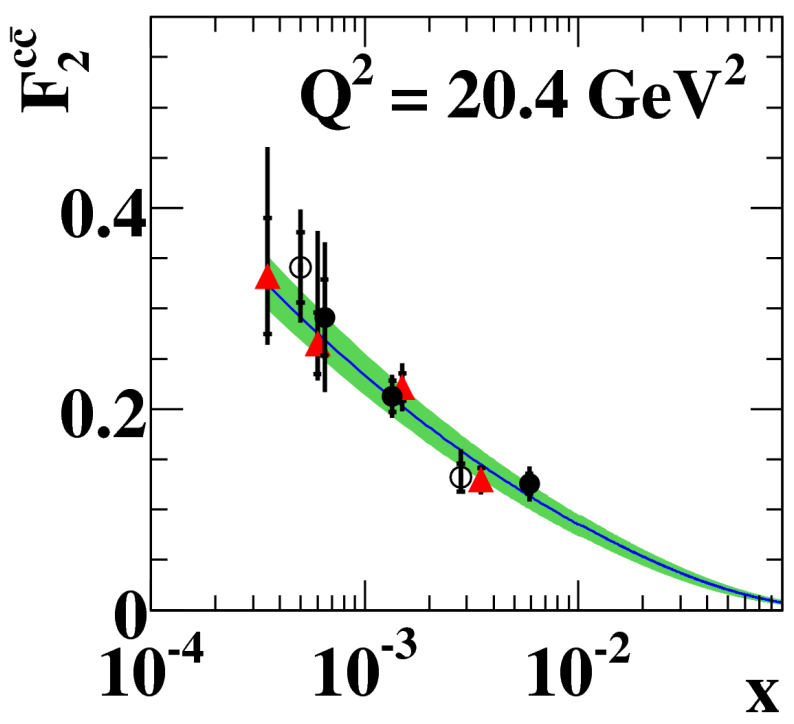

- $\quad$ ZEUS $\mathrm{D}^{ \pm}, \mathrm{D}^{\mathbf{0}}\left(133.6 \mathrm{pb}^{-1}\right)$

$\quad$ ZEUS $\mathrm{D}^{ \pm}\left(65 \mathrm{pb}^{-1}\right), \mathrm{D}^{0}, \mathrm{D}_{\mathrm{s}}^{ \pm}\left(82 \mathrm{pb}^{-1}\right)$

$\triangle \quad \operatorname{ZEUS~D}^{* \pm}\left(82 \mathbf{p b}^{-1}\right)$

\section{ZEUS NLO QCD}

- $\mathrm{F}_{2}^{\mathrm{cc}}$ from $\mathrm{D}^{ \pm}$and $\mathrm{D}^{0}$ were combined

- The results are more precise than a previous measurement using the same decays channels

- At high $\mathrm{Q}^{2}$ similar precision as $\mathrm{D}^{*}$

- The ZEUS NLO QCD fit describes the data well 


\section{$\mathrm{F}_{2}^{\mathrm{cc}}$ from muons}

\section{ZEUS}
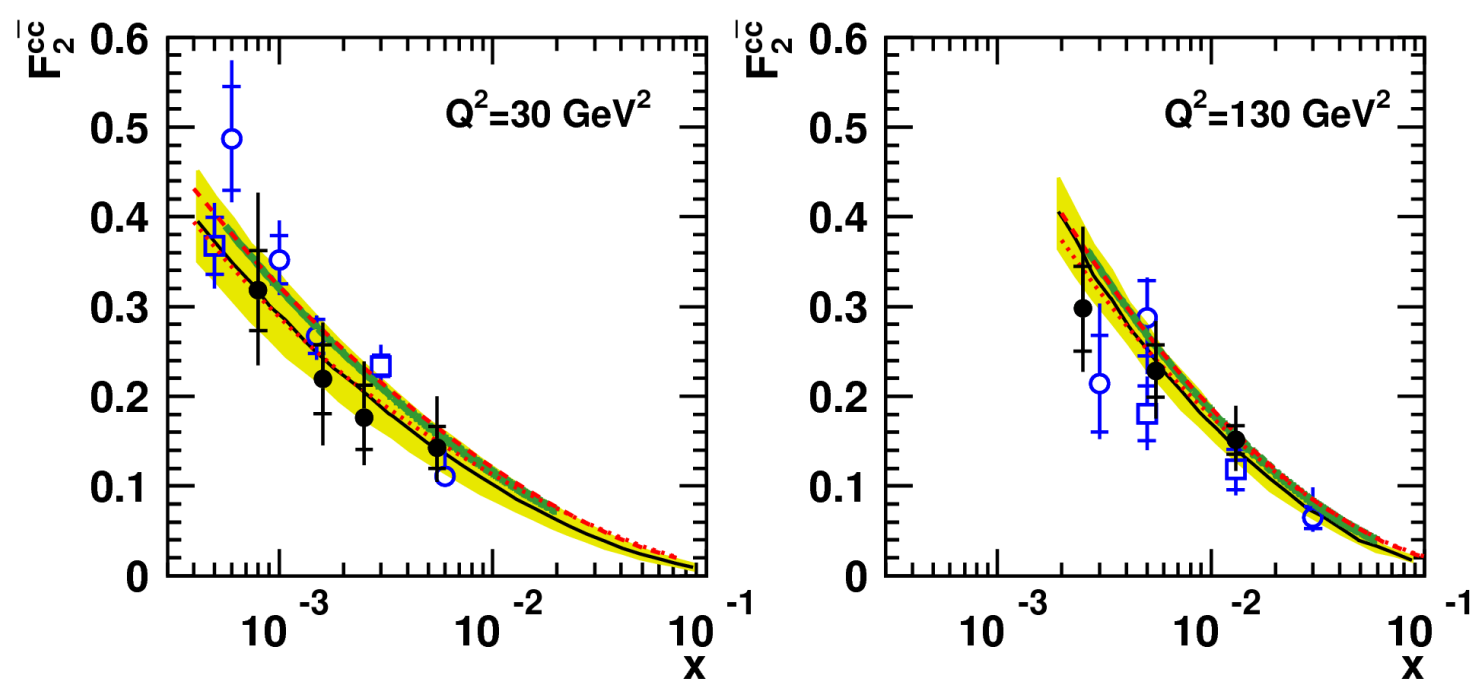

- The agreement between different charm tagging methods is good

- At high $\mathrm{Q}^{2}$ the precision of the presented data is similar or better than previous results

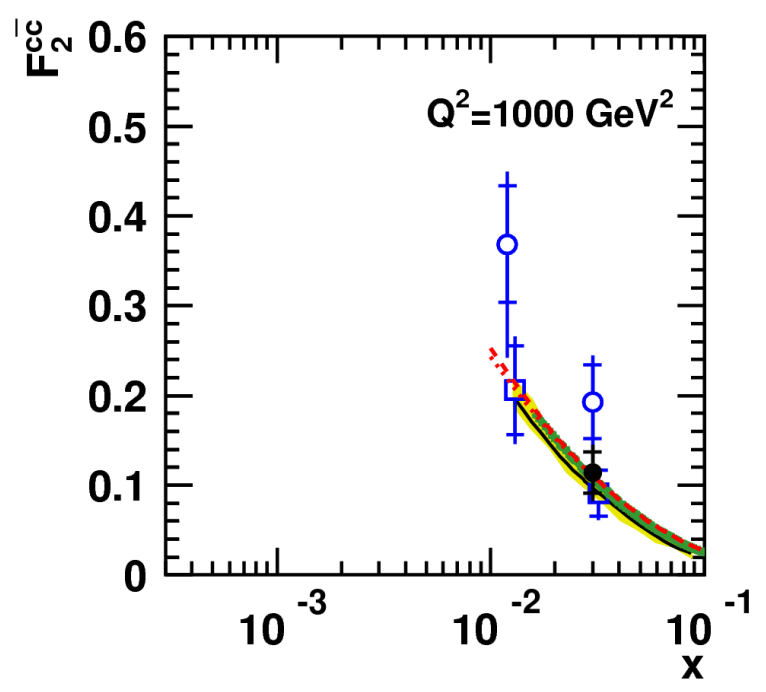

FFNS:

- ZEUS $126 \mathrm{pb}^{-1}(\mu)$

- ZEUS $82 \mathrm{pb}^{-1}\left(\mathrm{D}^{*}\right)$

$\mathrm{H} 157 \mathrm{pb}^{-1}$ (VTX)

$\square$ ZEUS-S FFNS

- CTEQ6.6

-. MSTW08 nlo MSTW08 nnlo
ZEUS-S

GJR08

GM-VFNS: $\quad$ MSTW08 NLO MSTW08 NNLO 


\section{$F_{2}^{\text {cc }}$ as a function of $\mathbf{Q}^{2}$}

\section{HERA}

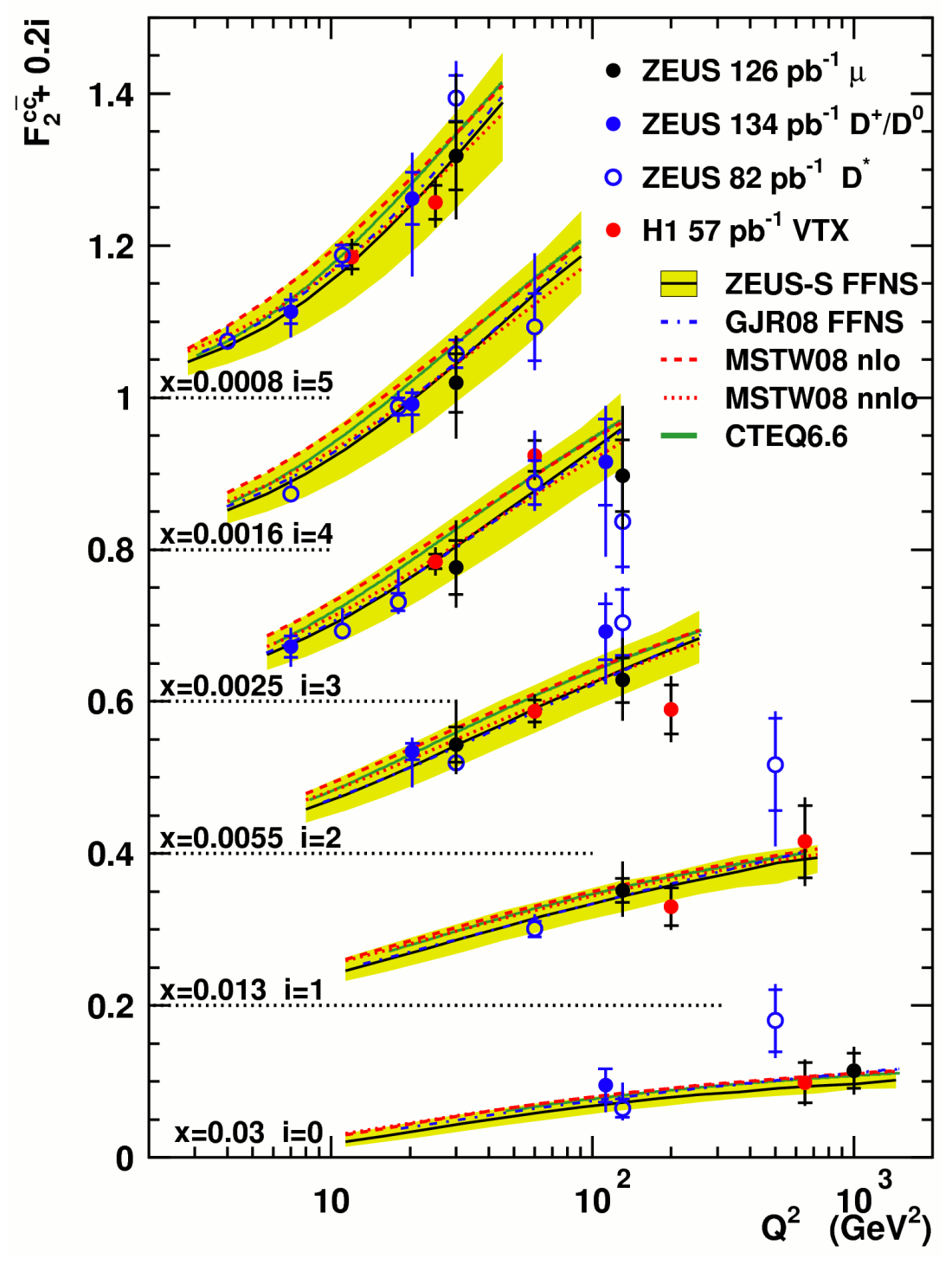

- The measurements are described by the shown QCD predictions

\author{
FFNS: \\ ZEUS-S \\ GJR08
}

GM-VFNS: $\quad$ MSTW08 NLO MSTW08 NNLO CTEQ6.6 


\section{Summary}

- Charm production has been measured using the decay channels $\mathrm{D}^{+} \rightarrow \mathrm{K}^{-} \pi^{+} \pi^{+}(+$c.c. $)$and $\mathrm{D}^{0} \rightarrow \mathrm{K}^{-} \pi^{+}(+$c.c. $)$and using semileptonic decays to muons

- Single differential cross sections are in good agreement with NLO QCD predictions from HVQDIS

- $\mathrm{F}_{2}^{\mathrm{cc}}$ was extracted and is in agreement with previous measurements and QCD predictions

- The precision will be improved further from the analysis of the full HERA II dataset $\left(\approx 350 \mathrm{pb}^{-1}\right)$ and by combination of different charm tags 


\section{Backup}




\section{Simulation of secondary vertices: $D^{0}$}
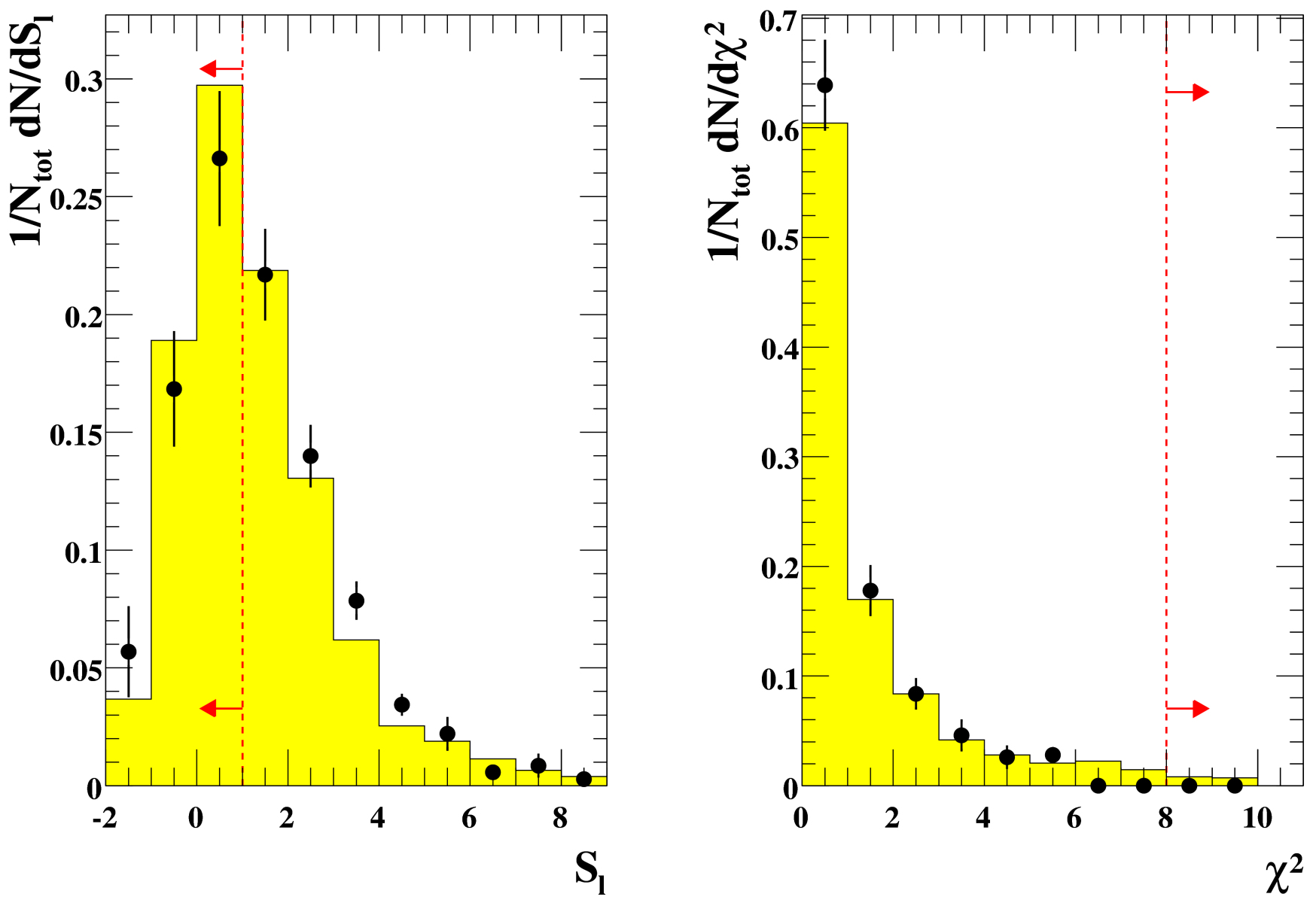


\section{PDF schemes and parameters}

PDF

ZEUS NLO

GJR08

CTEQ6.6

MSTW08 NLO

MSTW08 NNLO
Order

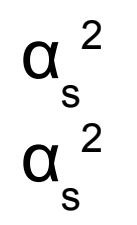

$\alpha_{s}^{2}$

$\alpha_{s}^{2}$
$\alpha_{s}^{3}$
Scheme

$\mu^{2}$

FFNS

FFNS

GM-VFNS

$Q^{2}+4 m^{2}$

$$
\mu_{F}^{2}=Q^{2}+m^{2}
$$

GM-VFNS $Q^{2}$

GM-VFNS $Q^{2}$
1.5

1.3

1.3

$m_{c}[\mathrm{GeV}] \quad m_{b}[\mathrm{GeV}]$

4.75

4.2

4.5

4.75

4.75 


\section{More predictions}

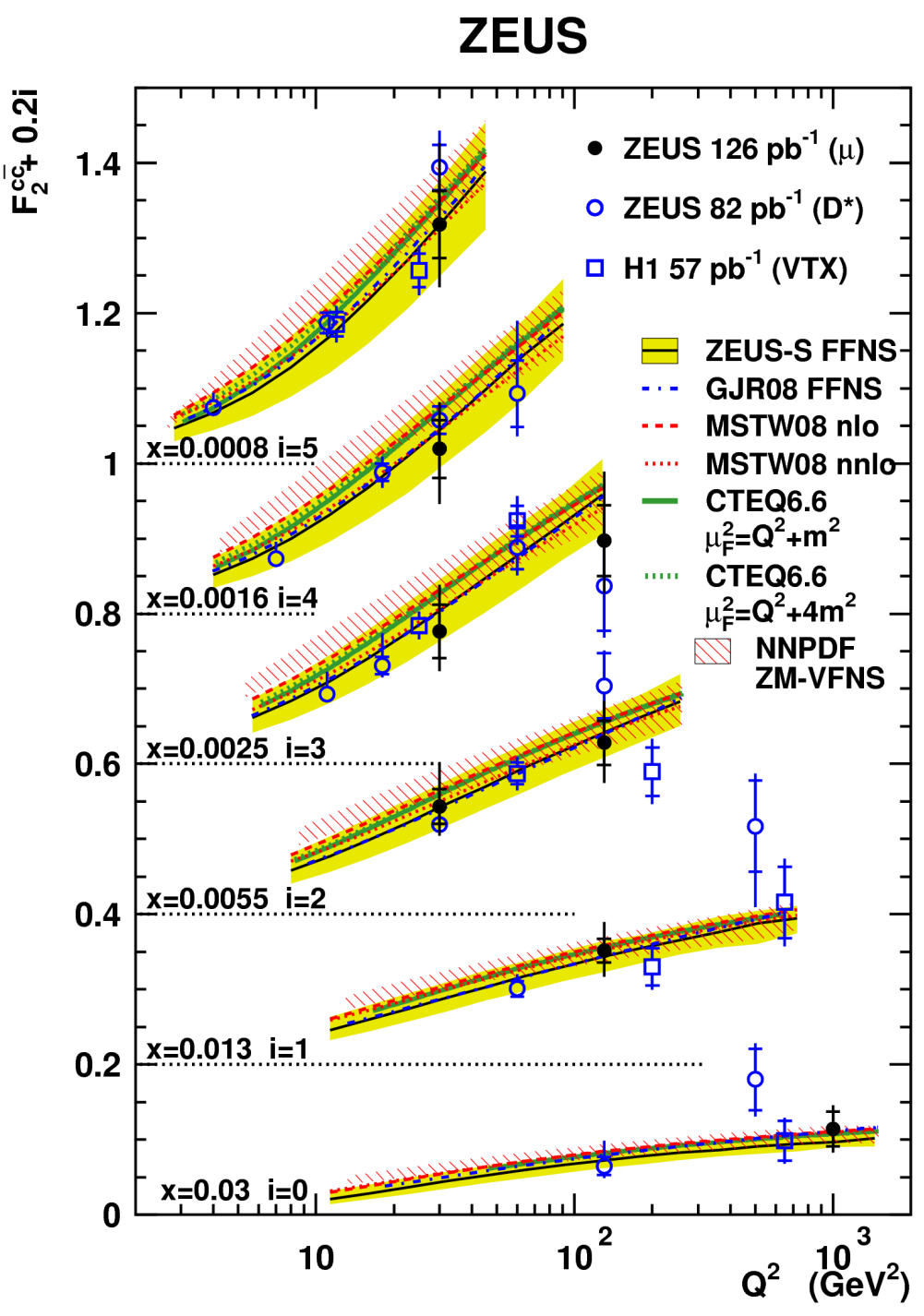

$\begin{array}{ll}\text { FFNS: } & \text { ZEUS-S } \\ & \text { GJR08 }\end{array}$

ZM-VFNS: NNPDF

GM-VFNS: CTEQ6.6

MSTW08 NLO MSTW08 NNLO 